\title{
el eongreso de Praga
}

FERNANDO AGUIRRE de YRAOLA, Dr. arquitecto

\section{Simapsîs}

En este artículo se comentan dos de los temas tratados en el Seminario de Praga, sobre la evolución de la estructura de la industria de la construcción, cuyos títulos son: $\mathrm{LLa}$ evolución de las relaciones entre las diversas personas que intervienen en la construcción, $y$ wha continuidad de la demanda $\mathrm{y}$ la continuidad técnica de la producción». Las ponencias que se glosaron estuvieron a cargo de los delegados de Dinamarca,
Polonia, Paises Bajos y Uerania. También fueron de gran utilidad para el estudio y la discusión de los interesantes puntos tratados, algunos documentos auxiliares redaetados $y$ comentados en las sesiones por delegados de otros países. El análisis del método de producción en cadena, o aFlow-line method», presenta especial interés.

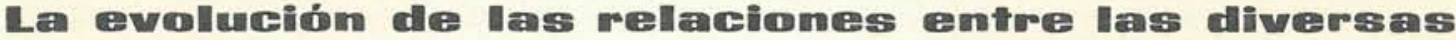

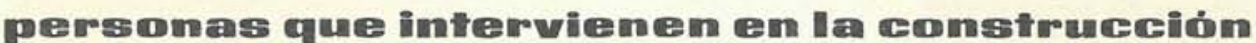

Continuando con la información del Congreso de Praga, cuya importancia para el futuro de la construcción ha sido señalada ya en números anteriores, nos proponemos en éste dar una referen cia de otros dos importantes temas tratados en el Seminario; son éstos: "Las modificaciones de la evolución de las relaciones entre las diversas personas que intervienen en la construcción» y "La continuidad de la demanda en la industria de la construcción».

El primer tema fue estudiado mediante las ponencias de los delegados Arctander y Bredsdorf, directores del Instituto Nacional de Investigaciones para la Edificación, de Copenhague, y la de Bógusz, de la Comisión para Planificación del Gobierno de Varsovia.

Ambas ponencias se han basado, parte en informaciones procedentes de las monografías nacionales de los países representados en el Seminario, y parte en conclusiones generales obtenidas por sus autores de las experiencias adquiridas en sus respectivos países, referentes a la adaptación del "equipo" que integran las personas que intervienen en la construcción a las exigencias de la producción industrial y del desarrollo de la industria de la construcción.

El problema, considerado desde el punto de vista escandinavo, es abordado mediante el análisis de las relaciones entre los miembros del equipo tradicional de la construcción. Este equipo, en realidad, constituye una organización provisional, establecida con el objeto limitado de dirigir, planificar y ejecutar cierto proyecto de edifício, situado en un lugar definido. Así, su tiempo de funcionamiento está limitado a la duración de una empresa o a la de una serie de empresas de construcción determinada.

Una de las características del equipo de construcción es la de que sus miembros pongan los medios técnicos y sociales que poseen, a la disposición, no sólo de un proyecto determinado para el que está formado el equipo, sino también-y al mismo tiempo-para otros muchos proyectos. Se comprueba, pues, que cada miembro del equipo puede ser independientemente miembro de otros distintos, hecho que, especialmente en los períodos de plena ocupación, puede causar dificultades considerables para la aportación a su debido tiempo de los medios necesarios para los diferentes proyectos y obras en los que está ocupado.

Se podría tal vez subrayar que las personas que asumen actualmente los cargos de miembros de un cierto equipo, no son necesariamente propietarios o directores generales de las empresas contratadas, sino solamente representantes con autoridad. Por este hecho, la relación fundamental 
entre los miembros del equipo se ha descrito de una forma muy apropiada como «autonomía independiente». Hay una falta de proporción entre la interdependencia técnica de los medios y la independencia desde el punto de vista de organización de los que les controlan.

Otra característica del equipo de construcción tradicional es que el modo de relación entre sus miembros no se establece según las reglas especificadas en los contratos cuando se efectúa el acuerdo con el cliente, sino más bien conforme a una repartición determinada de antemano de las responsabilidades $\mathrm{y}$ competencias funcionales que proceden de reglas establecidas por las diferentes asociaciones profesionales a las que pertenecen los miembros. Cada miembro conoce así su papel antes de comprometerse, pero como este papel se basa en la suposición previa de que la obra se realice por métodos tradicionales, resulta de esto una confusión considerable sobre la definición y reparto de las responsabilidades cuando el equipo tradicional está encargado de realizar obras según métodos no tradicionales. Por otra parte, las modificaciones del modo de relación que se imponen para la adaptación del equipo a los métodos industrializados pueden poner al miembro en una difícil situación con las costumbres y códigos profesionales.

Se presenta con esto una división aproximada en tres partes: cliente, equipo de planificación y equipo de construcción. Una de las características del equipo tradicional es que su contrato para la construcción se realiza muy tarde. Efectivamente, la práctica normal-prevista para las costumbres profesionales-tiende a aplazar el contrato del equipo de construcción hasta que las decisiones principales referentes a los planes materiales y métodos de construcción se hayan tomado por el cliente y el equipo proyectista. Esas decisiones se formulan en los proyectos, en los planos de obra y en las operaciones que tienen que efectuar los diversos contratistas especializados, así como en otros documentos que forman la base para las ofertas de los trabajos de construcción.

La ponencia danesa, una vez realizado este análisis, estudia las modificaciones que exigen una nueva organización del equipo de construcción.

En los nuevos sistemas industrializados habrá que satisfacer las exigencias de una producción técnica, $\mathrm{y}$, desde el principio de la elaboración del proyecto, el equipo de construcción deberá tomar las decisiones sobre los medios técnicos y elementos que deben disponerse y que tendrán que emplearse y producirse con objeto de llevar a cabo el proyecto de la forma más económica. Al no ser tradicionales las operaciones manuales y mecánicas en la obra, deben elaborarse y escribirse antes de la ejecución, y las actividades de la misma y entrega de los elementos prefabricados deben ser calculados minuciosamente para corresponder a los programas que dan las mejores posibilidades para las operaciones de rutina, las cuales tienen que establecerse y realizarse en series sucesivas. Esta nueva situación requiere una reorganización de las relaciones tradicionales entre los miembros del equipo de construcción.

Ya en el estado inicial del proyecto, los contratistas o especialistas de producciones técnicas deben estar comprendidos en el equipo con el fin de adaptar el proyecto a las exigencias técnicas y a la economía de la producción. Esta modificación que se refiere a la obra de la creación del equipo está en pugna con el sistema tradicional de adjudicación abierta; sin embargo, la modificación se ha realizado en muchos países. Esto solamente constituye una primera etapa en el cambio de las condiciones que deben seguirse por las modificaciones correspondientes del plan de construcción.

Una conclusión provisional de la parte que incumbe al equipo de construcción en el proceso de desarrollo puede resumirse de la siguiente forma:

Los equipos de construcción deben adaptar sus métodos y organizaciones según los desarrollos obtenidos en la iniciativa fuera del equipo. Esta solución presupone que los centros de desarrollo coordinados se establecen por otras instituciones permanentes (la industria de la construcción, organizaciones de clientes $u$ organismos gubernamentales).

Entre las finalidades de los equipos de construcción está la de planificar y elaborar ciclos de desarrollo con miras a mejorar la calidad de sus productos y favorecer los métodos de producción. Estos fines deben traducirse naturalmente en objetivos de operación determinados por la dirección del equipo.

Solución mixta de a) y b).

Para que los resultados obtenidos en el campo del desarrollo por los equipos de construcción puedan ser útiles para los centros de desarrollo y, por medio de éstos, para los equipos tradicionales de construcción, habrá que establecer un sistema de comunicaciones.

Probablemente la solución última es la que conviene mejor para hacer frente a las demandas de los usuarios, extendiéndose desde proyectos aislados hasta grandes series de proyectos.

Las teorías y principios de una organización industrial admiten generalmente como una cosa cierta el que una organización permanente única, una empresa comercial o un departamento gubernamental tenga el control absoluto de la concepción, desarrollo y producción del producto final. 
Construcción de vivien das, por métodos in dustrializados, en Ces. ké Budejovice (Checoslovaquia).

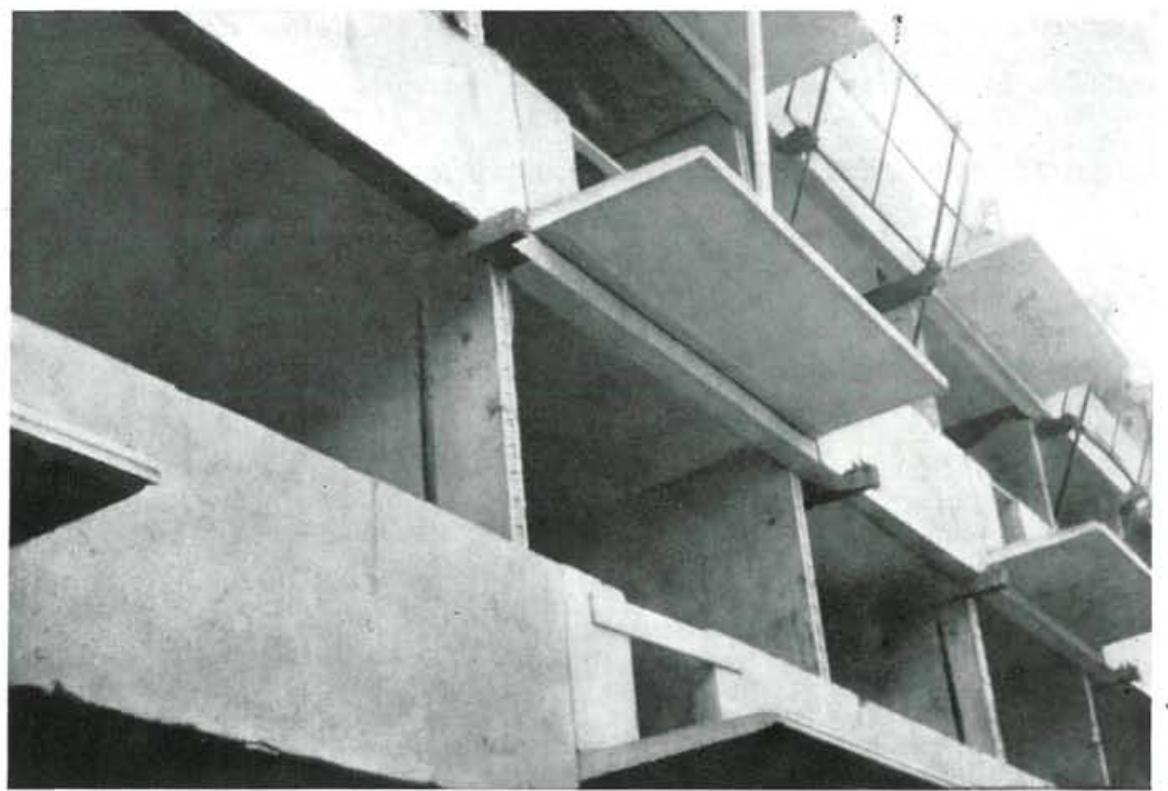

La dirección suprema de la organización comercial determina las finalidades a corto y largo plazo de la empresa; decide la política, programas de acción y conformidad de estas finalidades, toma las decisiones referentes a la subdivisión de la organización en unidades y el modo de relacionarlas entre sí, decide y atribuye las responsabilidades de la dirección y la salvaguarda de estas unidades, y nombra los dirigentes encargados de estas responsabilidades. La autoridad coordinadora se centraliza así, sin objeción, en la dirección suprema. Que el resultado sea una organización bien ordenada y sincronizada dependerá, entre otras cosas, de la calidad de la dirección y de los principios en los que se basan la concepción y los medios de trabajo del sistema de organización.

El modelo tradicional de la estructura de la organización, siempre empleado en muchas entidades comerciales, recomienda una división administrativa de la organización en unidades operativas: servicio de producción, servicio de control de ésta, servicio de ventas, servicio financiero, servicio de personal, etc. Los jefes que asumen la dirección de estos servicios son ayudados por un equipo (especialistas consejeros) bajo su jurisdicción.

\section{Equipo de comstrución melcutivos a los sistemens de prefaloricación "abiertos" o "cerredos"}

En el artículo publicado en el número anterior, que llevaba por título «El proceso de desarrollo...., introdujimos las nociones de los sistemas de prefabricación abiertos y cerrados. Recordando aquí dichos conceptos, designamos por sistemas "abiertos» a los basados en la producción en serie industrial de elementos funcionales cada vez más complejos, y que se prestan al montaje según combinaciones muy variadas y, por consiguiente, intercambiable en cierto grado. Según este criterio, los fabricantes venderán stocks de elementos constructivos, tales como paneles de fachada, losas y placas de forjado o tramos de escalera, es decir, elementos de un "Juego de construcción» que el contratista montará según los planos del arquitecto. Es lógico suponer que la producción según catálogo de elementos disponibles para el montaje será una de las características de la futura evolución de la industria de la construcción, base de la industrialización. Llamaremos sistemas "cerrados» a los métodos constructivos que exigen una unificación estricta extendida a las fases de los proyectos, a la prefabricación, al transporte y montaje de los elementos, y que se refieren, principalmente a la prefabricación pesada de grandes paneles.

Al utilizar estos conceptos en el tema de la "Evolución de las relaciones entre las distintas personas que intervienen en la construcción», resulta la necesidad de distinguir entre las características de los dos sistemas, o sea, del abierto y el cerrado. En las condiciones de prefabricación abiertas, las funciones importantes del estudio del desarrollo del proyecto, de la producción y del empleo de los elementos y de las partes prefabricadas del edificio son descentralizadas por los fabricantes 
independientes especializados en la construcción y en las otras industrias. Todos los elementos exis. ten en el mercado y pueden comprarse según catálogo. De esta forma, en el sistema abierto, el arquitecto ha conservado en el equipo de la construcción su tradicional libertad de elección, el ingeniero consultor está liberado de la carga de la producción de elementos y partes de la construcción, quedando a su cargo las operaciones de transporte, montaje y ensambladura de los elementos. El sistema abierto parece permitir la coordinación sin intervención económica.

Hay, por lo menos, tres condiciones necesarias para un desarrollo eficaz y coordinado del sistema abierto:

Las fábricas de elementos deben tener a su servicio muchos proyectistas, arquitectos e ingenieros.

Se debe establecer un sistema eficaz de comunicación entre el equipo de construcción y los fabricantes, con el fin de que las experiencias que obtengan los equipos de construcción se apliquen más adelante y se utilicen para un desarrollo ulterior de los elementos.

La responsabilidad del desarrollo y adaptación de los sistemas industrialmente extendidos de coordinación dimensional y determinación funcional de los elementos y métodos de enlace debe confiarse a las instituciones que acepte la industria. Debe añadirse una responsabilidad ulterior: el análisis de la repercusión que el desarrollo eventual de ciertos elementos tendría para el desarrollo de otros elementos y métodos de ensambladura. Los resultados de estos análisis deberían comunicarse a todos los fabricantes de elementos. Estas instituciones deberán también emplear arquitectos e ingenieros especializados en estas materias.

Una de las razones por las cuales se insiste tan firmemente en las anteriores condiciones, es que éstas demuestran que las reducciones de la carga de trabajo y responsabilidades del equipo de construcción responden a un fin y a una especialización de los recursos de la competencia técnica de la industria de la construcción.

Por otra parte, en las condiciones de un sistema de prefabricación cerrado (o más bien parcialmente cerrado, ya que ningún sistema perfectamente cerrado está en condiciones de funcionar) las funciones de la fabricación de elementos y su ensambladura en obra están fusionadas en una empresa. Este estado de cosas permite el establecimiento en el interior de una sola empresa con comunicación eficaz entre las operaciones en fábrica y en obra. Pero una parte de la libertad de elección tradicional del arquitecto se pierde cuando éste trabaja bajo las restricciones del sistema cerrado.

Existen muchos casos que muestran algunas características de los dos sistemas. Basándose en este breve estudio, parece completamente claro el modo de relación entre los miembros del equipo de construcción, que no puede estudiarse sin tener en cuenta el sistema normal de prefabricación.

\section{Trumstemencia de funciomes especialles a los miemblous - instituciones ajemos al equipo}

Presencia de un vigilante contable en calidad de planificador e inspector de costes, responsable de la elaboración de las relaciones de cantidades.

Establecimiento de Centros de Programación Electrónica, que colaboren a la confección de relaciones de cantidades y del programa de operaciones, abastecimientos, etc., en el proceso de construcción.

Esablecimientos de Oficinas de Planificación Técnica, que ayuden a los arquitectos y constructores en las planificaciones de producción, programación de operaciones en obra y confección de planos del terreno.

Establecimiento de Oficinas de Estudios de Trabajo, que presten ayuda en la planificación de las tareas y métodos de trabajo comprendiendo las operaciones manuales y mecánicas de la obra. 
Presencia del "Coordinador», agente especializado en la coordinación en obra de varias empresas (Francia).

Esteblecimientos de Centros de Alquiler de Máquinas y Equipo de Construcción, poniendo el material al servicio de los constructores (en algunos casos de los encargados de obra).

Existencias de "Grupos de Desarrollo», que elaboren el desarrollo del trabajo por cuenta de las organizaciones de los clientes y en colaboración con sus equipos de construcción. De esta forma, se descarga al equipo de construcción de una parte de su tarea.

Establecimientos de "Oficinas de Estudios de Construcciones-Tipo», que se encarguen de estudios de proyectos-tipo (con muchas variaciones) para fines especiales. La ayuda que ofrece comprende los diseños de construcción y de ensambladura, relaciones de cantidades, documentos que especifican las exigencias de calidad, etc.

Vamos, ahora, a estudiar el problema bajo el punto de vista de los países del Este, para lo cua analizaremos la ponencia del delegado polaco. También nos será útil considerar el trabajo presentado al Seminario por el delegado húngaro S. Keresztury, y que lleva por título "Problemas de coordinación de Proyectos Técnicos, Producción de Material de Edificación, Estructura de las distintas ramas de la industria de la construcción en la acividad de la edificación de viviendas».

Mediante estos documentos se puede adquirir un interesante conocimiento de los fenómenos que se manifiestan en los países socialistas.

La ponencia polaca enfoca el problema desde el punto de vista del proceso de las inversiones.

En la fase actual de desarrollo de las fuerzas productoras y de la especialización que trae consigo, numerosos organismos, que desarrollan actividades en distintos sectores, participan en el proceso de la inversión. Sin enumerarlos a todos se podrían citar como los más notables:

- Los órganos de planificación, que establecen, de acuerdo con los interesados, la amplitud de las inversiones.

- El jefe de la obra, que elabora el programa de la inversión en particular y controla el curso de su realización.

$\longrightarrow$ Los poderes dirigentes, que determinan las proporciones de los fondos de inversión acordados a los diferentes jefes de obra y aprueban sus programas.

- Los órganos encargados de la planificación de la distribución del terreno, que establecen, de acuerdo con el jefe de la obra, la localización de la construcción dada.

- Las oficinas de proyecto, que elaboran, según encargo individual del jefe de obra, la documenta. ción «individual» (proyectos y presupuestos), o sea, la documentación «tipo» encargada por las autoridades centrales.

一 Los órganos centrales y locales, que aprueban la documentación desde el punto de vista legal en lo que se refiere a la construcción y otras disposiciones concernientes a las inversiones.

- Las empresas de construcción, generales y especializadas, comprendiendo la base directa de los servicios, que realizan, de acuerdo con el plan económico nacional, las tareas confiadas por el jefe de obra.

- Los Bancos, que financian las inversiones y ejercen el control a este título.

- La vigilancia de la construcción por el Estado, que controla, independientemente del jefe de la obra, los trabajos desde el punto de vista de la aplicación de las reglas legales.

- El fabricante de materiales y elementos de construcción.

- Los Institutos de Investigación y los Laboratorios de la Construcción, que estudian los problemas del progreso técnico y de la economía de las inversiones, así como los centros de información, que popularizan los mejores proyectos y materiales.

- Los órganos locales y centrales, que coordinan la actividad de los distintos participantes en lo que se refiere al proceso de la inversión. 
El abandono progresivo de los métodos tradicionales y la adopción de los métodos industriales de organización de la producción aportan cambios notorios en el sistema tradicional de relaciones entre los participantes en el proceso de la inversión.

Las experiencias prueban que la industrialización de la construcción influye sobre el establecimiento de los planes. Las decisiones referentes a la industrialización de la construcción entrañan, por regla general, un aumento de fondos de inversión necesarios para organizar el abastecimiento de los materiales.

Estas decisiones no pueden ser tomadas sin saber si este aumento de fondos es suficientemente motivado por la amplitud de la construcción prevista y el período de su realización. Es necesario, por consiguiente, que las de cisiones de la planificación de terminen la amplitud y la localización de las construcciones, así como su puesta en obra. Los planes económicos a largo plazo, que en los países socialistas es tablecen las perspectivas de desarrollo de la economía nacional, sirven de base para precisar los métodos de construcción en cier tas regiones determinadas del país. Es, pues, sobre la base de estos planes, donde se determinan las localizaciones de la construcción industrializada, la localización y la extensión de la fabricación de materiales y de elementos de construcción, así como el potencial de empresas de construcción especializadas en este dominio. La prefabricación de elementos ligeros o de elementos que pueden ser transportados sin dificultad a grandes distancias no está ligada estrechamente a unas regiones estrictamente determinadas, pero la prefabricación de elementos pesados cuyos transportes ocasionan grandes dificultades debe ser adaptada al plan de construcciones que estos elementos determinan.

La cooperación entre los organismos de planificación y las organizaciones de jefes de obra, con las oficinas de proyectos y las empresas de construcción, debe ser más íntima. Las decisiones concernientes a la aplicación de métodos adecuados en regiones determinadas, limitan la elección de la tecnología.

Las observaciones prueban que la transferencia de los trabajos de obra a las empresas industriales auxiliares o especializadas es una de las principales corrientes del progreso técnico. Sin precisar las causas de este fenómeno, conviene resaltar que influye eminentemente, tanto en la estructura de las empresas de construcción como en sus relaciones con las fábricas de materiales y elementos de construcción. Se puede observar igualmente, que el número de ramos de la industria, cuya producción está destinada a la construcción, aumenta constantemente. Además de los ramos industriales tradicionales de la construcción, tales como el cemento, la madera, el vidrio, los productos cerámicos, etc., nuevos ramos de la industria de productos químicos, electrotécnicos, de aluminio y de acero, vienen a aumentar la gama de materiales de construcción. El número de organizaciones de producción que participan en el proceso de la construcción aumenta. 
Este proceso se acompaña de ciertas modificaciones en la organización de las empresas de construcción. Por un lado existe la tendencia a agrupar sus actividades en un solo órgano que se manifiesta por la creación de grupos de empresas especializadas en ciertos tipos de construcción (construcción de viviendas, de escuelas, hospitales, centrales eléctricas, etc.). Estos grupos reúnen, en una sola organización, a las fábricas de materiales y de elementos de construcción y a las empresas de diversas especializaciones. Por otra parte, se tiende a la especialización muy intensa de producción de materiales para los elementos de construcción, a la especialización de ciertos trabajos o a la ejecución de ciertas partes de las obras (montaje de estructuras de acero, instalaciones sanitarias y eléctricas, trabajos de nivelaciones, de aislamiento, de colocado de cimientos, de enlucidos, etcétera).

Las experiencias de varios países nos han permitido aceptar algunas conclusiones obtenidas de la práctica. Las monografías nacionales de la Unión Soviética y de Checoslovaquia nos muestran que este tipo de agrupaciones tienen sus buenas cualidades y sus defectos. Entre las buenas cualidades de estas agrupaciones figura la de que ellas son más independientes y que en la fase actual de desarrollo, es más sencilla y ligera la cooperación entre los distintos sectores. La reunión de varias funciones distintas en una agrupación garantiza el ritmo del proceso tecnológico-desde la producción de los elementos hasta su puesta en obra-. Esto permite acortar el ciclo de la construcción. Siguiendo las opiniones enunciadas en la monografía soviética, las agrupaciones de empresas especializadas en la construcción de grandes paneles han triunfado completamente.

Se observa una tendencia a intensificar la cooperación entre las oficinas de proyectos y las empresas de construcción, a medida que se desarrolla la industrialización de la construcción. Esta tendencia se manifiesta muy diversamente. Existen a veces pequeñas oficinas de proyectos organizadas al lado de las agrupaciones de empresas especializadas, que no elaboran más que detalles o que adaptan el proyecto general presentado por el jefe de la obra a las exigencias y posibilidades de la em. presa dada. Estas pequeñas oficinas podrían compararse a los despachos de construcción que existen junto a las empresas industriales.

Las empresas de la construcción industrializada tienden en general a que el conjunto del proyecto sea realizado por las oficinas de proyecto que le están subordinadas, considerando que esto facilita al autor del proyecto una mejor orientación en las posibilidades tecnológicas de la empresa de construcción, y permite a esta última reforzar su actividad.

Este importante problema se ha discutido extensamente; varios especialistas están de acuerdo en que la subordinación completa de las oficinas de proyectos a las empresas de construcción, a pesar de sus aspectos positivos, debilita sensiblemente al mismo tiempo la posición del jefe de obra y crea la prioridad de los problemas tecnológicos sobre la calidad de las soluciones de los proyectos, desde el punto de vista funcional y económico.

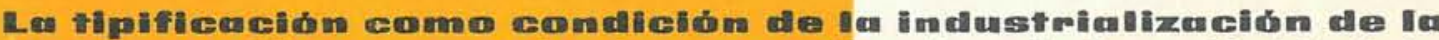

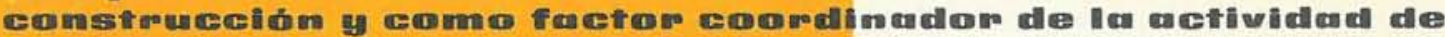

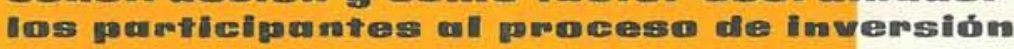

Una de las pruebas de la importancia para el desarrollo de la industrialización que representa la tipificación de elementos, es la aparición y análisis de este concepto en casi todos los temas en los que se dividió el estudio del Seminario.

En el número anterior ya introdujimos unas ideas sobre la imperiosa necesidad de poner a punto en toda Europa los estudios sobre tipificación y coordinación modular.

La demanda en masa de inmuebles y de elementos, homogénea en el fondo, y el proceso de industrialización de la construcción reclaman la coordinación de las actividades de los diferentes participantes al proceso de la inversión. La tipificación propiamente dicha como principio para el establecimiento del proyecto, de los elementos, así como de los métodos de construcción, son una de las formas más esenciales de esta coordinación. La tipificación así concebida, constituye un campo nuevo de la actividad que resulta de la industrialización de la construcción. La tipificación en esta industria es la resultante de las necesidades y de las posibilidades de las fábricas de elementos prefabricados, de las empresas de construcción y de los futuros usuarios. Regula y limita las exigencias individualizadas. Esta limitación está compensada por una mejor satisfacción de las necesidades, gracias a la aplicación de métodos de producción industriales cuyo rendimiento es mayor.

Como la tipificación es la resultante de intereses distintos, las decisiones sobre la misma son adoptadas por las autoridades centrales y no por los distintos participantes en el proceso de la inversión. 
En la mayor parte de los países las decisiones en materia de tipificación proceden siempre de los órganos gubernamentales llamados a coordinar la actividad de la construcción.

La tipificación estabiliza, en cierta manera, las soluciones para un período determinado. Ella permite aplicarlas en masa y fabricar los elementos en grandes series, y no está justificada más que por el orden del tamaño de las unidades y, sobre todo, por la repetición. La cantidad y el tamaño de los edificios adicionales (establecimientos de cultura y de educación, edificios sociales, así como de abastecimiento) están fijados por normas distintas, según el orden y extensión de la unidad vecinal, barrio residencial, etc., teniendo en cuenta el numero de habitantes y las distintas circunstancias demográficas.

La disposición de los edificios, el grado de concentración de las viviendas, la centralización de los edificios industriales en grandes unidades compactas, y la dispersión de los edificios agrícolas dificilmente accesible exigen métodos diversos de producción y de realización y, por lo tanto, también de tipificación.

Para que la estabilización no frene el proceso técnico, es indispensable preparar un nuevo impulso hacia las soluciones funcionalmente mejores y más eficaces. La experimentación y las soluciones de prototipo toman así una importancia nueva. Como la tipificación es la resultante de las aspiraciones de los participantes en el proceso de inversión, la experimentación y los prototipos que preparan la etapa sucesiva necesitan la cooperación de todos los interesados. Resulta de lo que precede, que la coordinación de la actividad de los jefes de obra, de los autores de los proyectos, de las empresas de construcción, de las fábricas de materiales y de elementos de construcción, de los institutos de investigación y otros participantes en el proceso de la inversión adquiere importancia como consecuencia de la industrialización de la construcción.

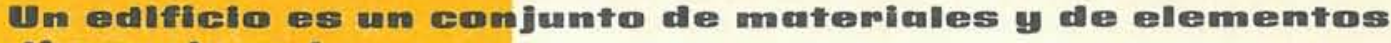

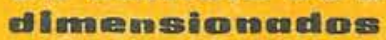

Se ha hecho indispensable establecer una clasificación: situar cada elemento dimensionado en su lugar, precisar las relaciones de este elemento con los otros y, en fin, definir el sentido exacto de la palabra que designa a este elemento. Por una parte, una clara distinción entre materiales y elementos dimensionados permite separar los trabajos que pueden ser industrializados de los que se realizan necesariamente en la obra. Esta distinción recae sobre la concepción misma del edificio. Cada empresa y taller puede concentrar su esfuerzo de producción en ciertos productos dados y mejorar así la calidad, las condiciones económicas de producción y la distribución. Cuando se utilizan materiales y elementos dimensionados prefabricados, los trabajos de obra exigen menor número de operarios y su carácter se modifica. Se pasa de la ejecución tradicional «in situ» al simple montaje. La concepción misma del edificio evoluciona paralelamente, desde el edificio homogéneo que comprende diversas partes hechas bajo medida que no pueden ser industrializadas hasta el edificio constituido por un cierto número de elementos predeterminados que se les ensambla de acuerdo con los planos.

Cuando se trata de fabricar materiales y elementos modulares, será de gran utilidad tener en cuenta las recomendaciones publicadas por la Agencia Europea de Productividad de la Organización Europea de Cooperación Económica, que indican características muy importantes para la definición de elementos modulares.

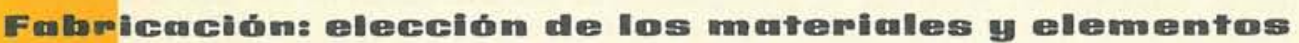 moduleares}

Los materiales y elementos modulares pueden distinguirse de los habituales dimensionados, en dos puntos principales: deben ser dimensionados en función de un espacio modular standard; su perfil debe permitir el ajuste de los materiales y elementos adyacentes. En consecuencia, puede ser necesario modificar al mismo tiempo la forma y las cotas de los materiales y elementos existentes. El género y la importancia de las modificaciones aportadas a las cotas y perfiles dependerán de la utilización prevista y de las relaciones que se establezcan entre los materiales y elementos considerados, y también entre los otros elementos que entran en la construcción. 


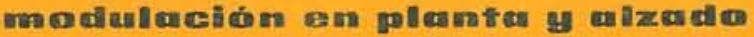

(Rochester Temple School).
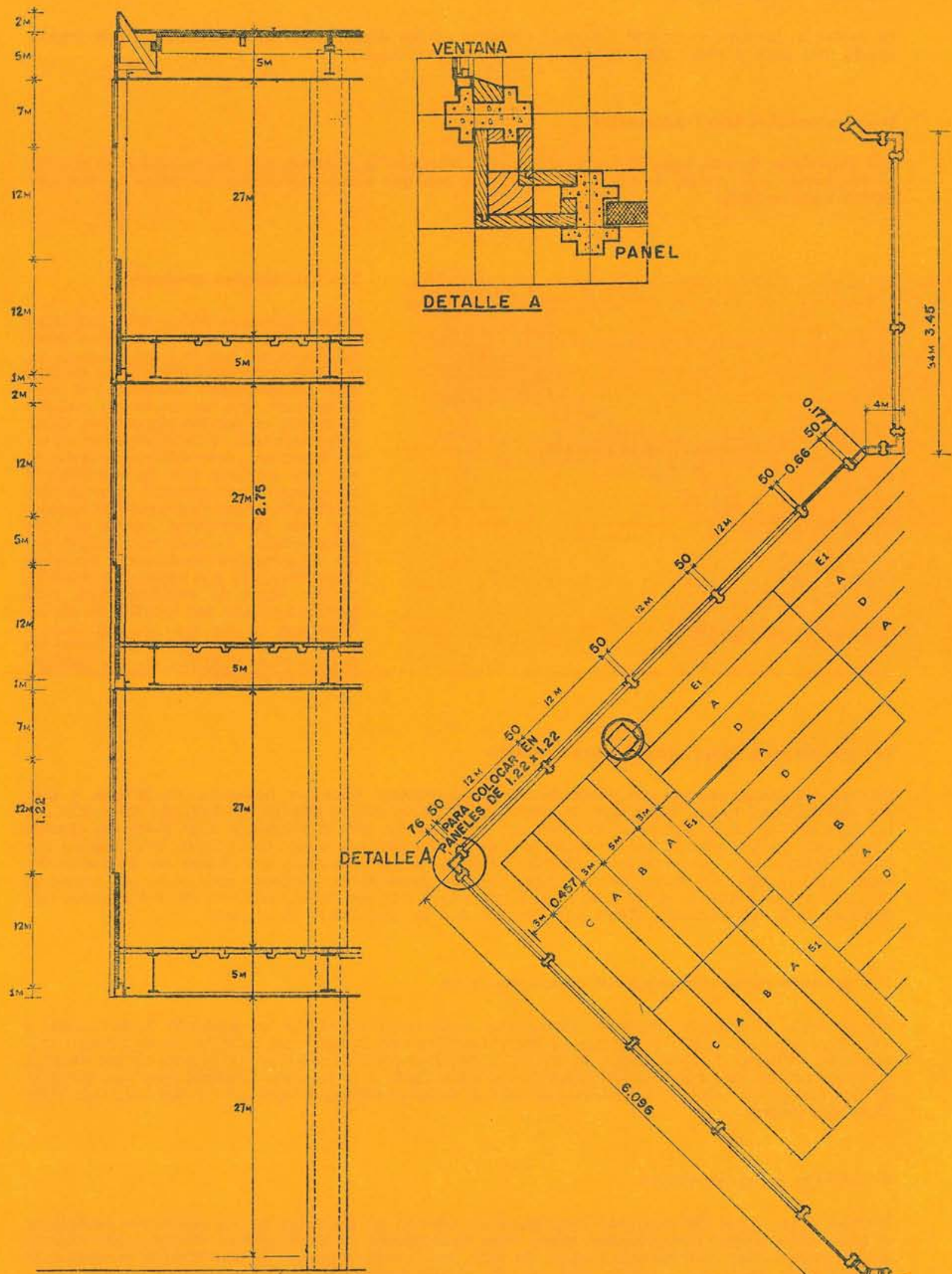

TALLEA
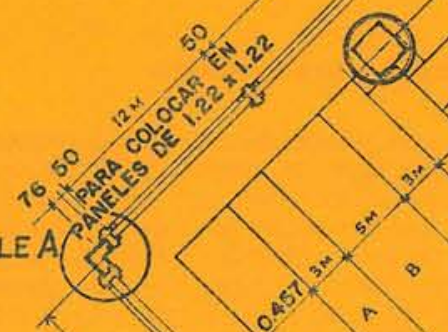


\section{Los materiales amorfos}

Es evidente que en este primer grupo la cuestión de las dimensiones no presenta ninguna importancia real bajo el punto de vista de la coordinación modular.

\section{Los materiales semi-acabados}

Los materiales de este tipo, llamados igualmente secciones o perfiles, son normalmente productos en longitudes indefinidas, de tal forma que basta con que estén moduladas las cotas de sus secciones transversales.

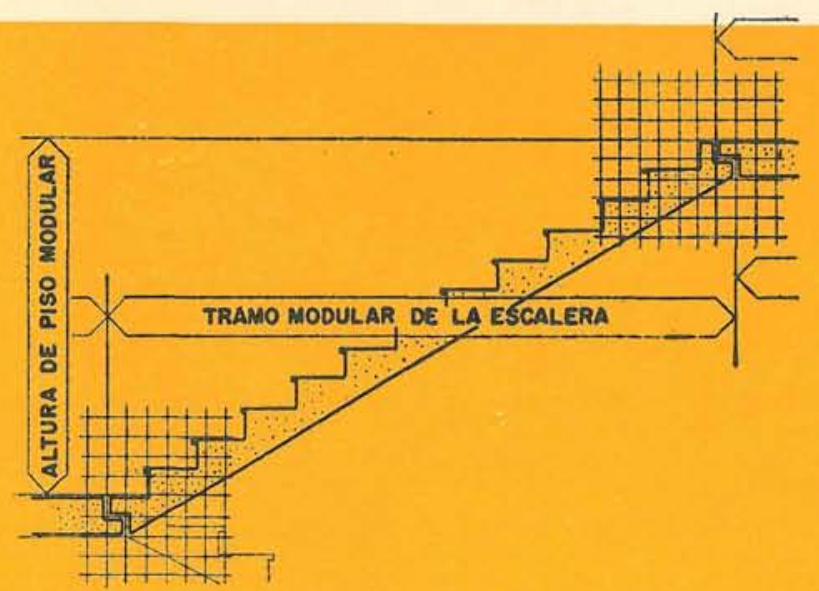

ESCALERA MODULAR

\section{Los materiales acabados}

Estos materiales tienen cotas reducidas en las tres dimensiones y es, en general, suficiente que un cierto número de unidades colocadas una al lado de otra den una dimensión modular. Tan cierto es esto, que en un cierto número de países es posible alguna vez hacer construcciones en ladrillos teniendo cotas modulares determinadas, a pesar de que los ladrillos que las constituyen no tengan forzosamente dimensiones moduladas. Cuando se trata de elementos más importantes, como son los aglomerados, es necesario conferirles las dimensiones modulares. Podemos llegar también a modular las cotas de ciertos tipos de canalizaciones, más particularmente sus longitudes, sin olvidar que en este caso los empalmes y juntas tienen una influencia sobre las dimensiones efectivas de las canaliza. ciones en cuestión.

\section{Los elemenfos complejos de construcción}

Se trata de elementos que son particularmente interesantes para ser obtenidos en fábrica, y que, por consiguiente, temen estar estrechamente coordinados con el cuadro modular de referencia. La mayoría de los casos, sus cotas deberán ser dadas en las tres dimensiones. Casi todos los elementos de este grupo deberán ser acotados en función del emplazamiento que ocupen en el cuadro modular Solamente a este precio se podrán coordinar estos elementos que deben ser yuxtapuestos, como, por ejemplo, los armarios de cocina. De esta manera, un elemento aislado no se extenderá más del espacio que le es asignado en el plano y podrá ser coordinado con otros elementos funcionales de la construcción, como, por ejemplo, con las paredes o con el piso.

\section{Los elementos funcionales de la consfrucción}

Por la razón de que los elementos funcionales están constituidos por un conjunto de materiales y elementos que han sido dimensionados individualmente en función del módulo, no es preciso aplicarles un esfuerzo de modulación específica. A pesar de todo, debe indicarse, aunque no sea esencial, la necesidad de coordinar cuidadosamente las estructuras y el plan de canalizaciones con los principales cuadros de referencias utilizados para el establecimiento del proyecto y con las partes esenciales del edificio.

\section{El edificio en su conjunto}

Incluso si las cotas del conjunto resultan de la adición de las cotas de los elementos modulares, el proyectista tendrá libre elección sobre estas dimensiones. Notemos que en ciertos casos vendrán determinadas estas condiciones, a pesar de todo, por la cota standard de un elemento de dimensión considerable, como, por ejemplo, una casa de campo normalizada. 


\section{el congreso de Praga \\ FERNANDO AGUIRRE de YRAOLA, Dr. arquitecto}

\section{La comeînuidad cle la demanda

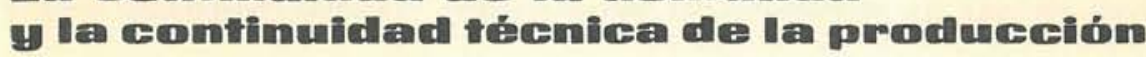

El tercer tema específico del Seminario de Praga fue glosado por las ponencias del delegado de los Países Bajos, A. Joolen, director general adjunto del Ministerio de la Vivienda de su país, y por $\mathrm{N}$. Kanuka, director adjunto del Instituto de Investigaciones Científicas de la Industria de la Construcción de Kiev.

El contrapunto de los dos enfoques sirvió para analizar este problema capital para el futuro de la edificación según las ponencias citadas, que se desarrollan cínéndose a los siguientes puntos:

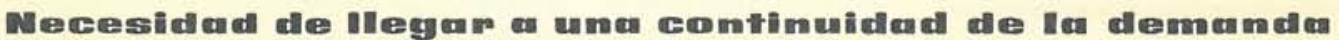

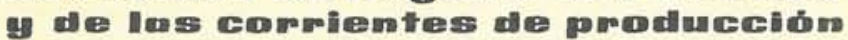

En la mayor parte, si no es en la totalidad de los países europeos, muchos factores crean un desequilibrio entre la demanda y la oferta de capacidad de construcción, excediendo la primera a la segunda.

Entre los más importantes se pueden citar:

Por parte de la demanda: Los daños causados al patrimonio inmobiliario durante los años de guerra-1939-1945-, que han creado una demanda enorme de trabajos de reconstrucción; el impulso demográfico de los últimos años, que ha aumentado considerablemente la demanda de viviendas; la creciente prosperidad, que ha acentuado esta tendencia cuantitativa y cualitativamente.

lib Por parte de la oferta: Durante la segunda guerra mundial y los primeros años que la han seguido, la actividad de la industria de la construcción ha sido muy débil y a veces nula; muy a menudo, la falta de mano de obra impide que la oferta se desarrolle al ritmo deseado. A esta dificultad se une el que ciertos gremios relacionados con la construcción han perdido su eficacia; la escasez de materiales de construcción en los primeros años de la postguerra han estan. cado el desarrollo de la industria de la construcción. 
Es necesario, pues, intentar una continuidad entre las corrientes de la producción, lo cual solamen. te es posible cuando existe paralelamente una continuidad en la demanda.

\section{Interdependencia de la continuidad de la demanda y de la continuidad de las corrienfes de producción}

Si la demanda sobre el mercado aumenta o cisminuye irregularmente, resulta una incertidumbre en cuanto a la capacidad de construcción necesaria que crea el riesgo de comprometer la aplicación de las medidas propias para asegurar una continuidad a las corrientes de producción. Por otra parte, la continuidad de la demanda trae consigo la continuidad de la oferta, es decir, de las corrientes de producción.

Si se produce una discontinuidad en el desarrollo de uno de estos elementos, resultan tensiones que crean una discontinuidad en el otro. Esta interdependencia entre la oferta y la demanda es debida, principalmente, a la imposibilidad de "almacenar» la capacidad de construcción.

\section{Continuidad de la demanda.-Méfodo estadístico de estudio}

Como consecuencia de lo expuesto anteriormente, es importante conocer las necesidades futuras para poder evaluar notablemente la demanda a la cual la industria deberá responder. Dada la diversidad de métodos de estimación, a menudo condicionados ellos mismos por los datos estadísticos de que se dispone, hay que establecer estimaciones distintas para cada sector particular de la industria de la construcción. Para evaluar las necesidades en materia de viviendas se usan, generalmente, los datos establecidos por los censos de población y de la vivienda, y los que se poseen sobre las variaciones del patrimonio inmobiliario y de la evolución de las cifras de población. Se pueden también estimar las necesidades del sector público (escuelas, hospitales, etc.), a partir de los datos obtenidos de los censos de población.

\section{Ajuste de la corriente de la demanda a la corriente de la producción}

Dado que la demanda de construcción es superior a la oferta, existe un riesgo de tensión. Si ésta se produce, resultará indefectiblemente un aumento de los precios acompañado, sin duda, de una baja en la calidad del trabajo. Hay muchas probabilidades para que este desequilibrio del mercado de la mano de obra traiga consigo una disminución de la productividad del trabajo. Es necesario, pues, impedir que el desequilibrio entre la oferta y la demanda repercuta en el mercado de la construcción y, en consecuencia, la demanda efectiva se ha de adaptar a la capacidad de construcción de la oferta, que ha sido explotada al máximo; en otras palabras, es necesario liberar al mercado de una parte de la demanda que ejerce presión sobre la capacidad de construcción.

\section{Continuidad técnica de la producción.-Método estadístico de esfudio}

Es superfluo subrayar cuán necesario es conocer, con toda la precisión necesaria, la capacidad fu. tura de construcción. Lo mismo que para las necesidades futuras, también es preciso hacer aproximaciones a largo plazo, a plazo medio y a corto plazo. Por otra parte, no es menos importante evaluar las componentes de la capacidad de construcción que evaluar el conjunto. Como la industria de la construcción exige, generalmente, una gran aportación de mano de obra, se empezará por las estimaciones de esta mano de obra, que serán seguidas por la estimación de la probable importan. cia de los gastos de inversión. Es necesario también establecer una distinción entre los sectores de la inđustria de la construcción según su carácter particular: obras de ingeniería civil, construcción de viviendas, construcción de fábricas y de talleres, etc. Es indispensable conocer la importancia de estos sectores para llegar a un ajuste preciso de la demanda.

\section{Organización de una produceión continua}

Este título trae a luz el planteo del siguiente problema: ¿Cómo hacer para que la producción de la industria de la construcción llegue a responder a las necesidades de la demanda? Hay distintas formas de solucionar este problema: acelerar el ritmo de la productividad, aumentar el efectivo de 
Cercos de paneles, fabricados en serie, en la ractoria para prefabricación de grandes panes en

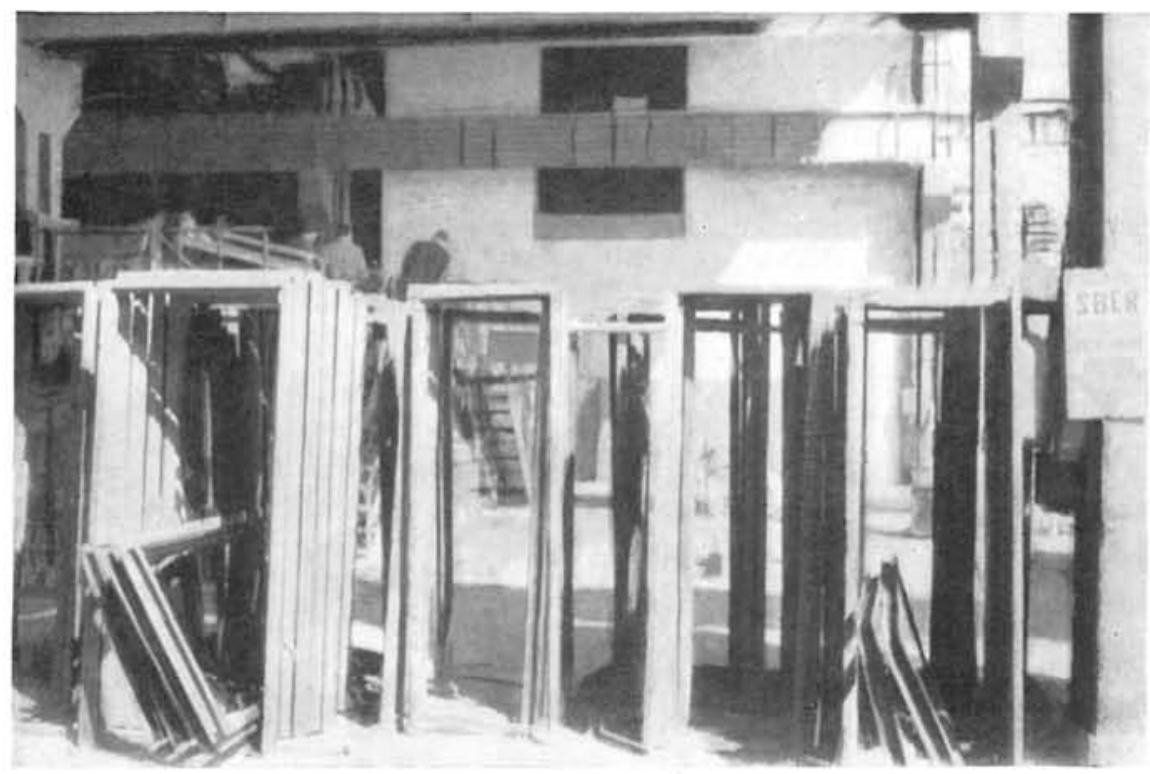

los trabajadores mejorando su estatuto social y alentando la formación profesional, impulsar la industrialización del proceso de la construcción, estimular la continuidad, etc. Esta última posibilidad es importante; en efecto, si no existe continuidad, una parte de la capacidad de construcción es desaprovechada. Conviene subrayar, a este respecto, la necesidad de una planificación juiciosa, que permita coordinar las actividades de todos los participantes a la operación de la construcción (cliente, arquitecto y constructor) y que englobe todas las fases del proceso de ésta. Será necesario, también, coordinar la ejecución de un gran número de conjuntos, para que la unidad de producción, una vez terminados los trabajos en una obra, sea transferida inmediatamente a otra.

Los trabajos de la nueva obra deberán estar en un estado que permita a la unidad de producción ponerse en funcionamiento desde su llegada. Se habrá realizado íntegramente la continuidad cuando se haya llegado a un encadenamiento análogo para todas las unidades de producción. El grado máximo de continuidad al que se puede llegar dependerá mucho de la uniformidad de los trabajos. A este respecto, la proporción en que los trabajos de construcción se hayan podido transferir de la obra a la fábrica, es decir, el provecho que se haya sacado de los elementos prefabricados, es también un factor importante. Cuantos más modelos haya, los elementos y materiales de construcción serán más diversos $\mathrm{y}$, por lo tanto, más difícil será ajustar continuamente la demanda a la oferta de capacidad de construcción.

En los países de Europa Occidental, la continuidad de la producción de la industria de la construcción depende, en gran parte, del mercado y de la voluntad de los empresarios individuales, y se encuentra, frecuentemente, con dificultades por una ausencia de demanda suficiente. Este problema no se presenta en los países socialistas. Aquí, la continuidad de prođucción y el ritmo de su desarrollo están previstos en los planes estatales relativos a la construcción, la cual representa una parte sumamente importante de la economía nacional del país. No hay ninguna duda de que una gran utilización de los proyectos-tipo, la práctica de construcciones en grandes series, la uniformidad de los elementos de la construcción, y la normalización y la coordinación modular, son muy importantes para asegurar la continuidad de la producción, sobre todo cuando estas medidas están tomadas a escala nacional. En numerosos países, su aplicación está dificultada por la ausencia de coordinación entre las actividades de diferentes empresas individuales.

En Checoslovaquia, en Polonia, en la República de Ucrania y en otros países socialistas, la utilización de los proyectos-tipo, el empleo de un único módulo y la uniformidad de las estructuras y de los elementos de construcción son obligatorios y muy ampliamente extendidos.

La construcción experimental, sin la cual es imposible mejorar constantemente los proyectos de vi. viendas, no se beneficia siempre del sostén necesario que deberían aportar los gobiernos de los países de Europa Occidental. Las diversas municipalidades y sociedades de construcción actúan 
independientemente de las construcciones experimentales; la aprobación de los poderes públicos interviene solamente cuando se obtienen resultados positivos.

Numerosos países se interesan mucho en el carácter reiterativo de las operaciones constitutivas del proceso de la construcción y en la introducción de los métodos de trabajo en cadena. No obstante, a pesar de toda su importancia, éste no es el problema esencial y decisivo. El sistema más eficaz consiste en construir edificios según un flujo tecnológico continuo de larga duración, como ocurre en Checoslovaquia, por ejemplo, en donde cerca de un 70 por 100 del volumen total de la construcción de viviendas está realizado por métodos de trabajo en cadena, y lo mismo ocurre en la República de Ucrania.

El proceso global de la construcción de un edificio se considera como una combinación sistemática de los procesos de aprovisionamiento, transporte y montaje. Los procesos de montaje y colocación que se efectúan en obra presentan una importancia muy particular; de su ritmo depende la alinea. ción de los otros procesos. A cada tipo de edificios de viviendas le corresponde una cierta tecnología, que puede estar representada en forma de normas tecnológicas. Estas son documentos básicos para los puestos de trabajo tipo, englobando una o dos unidades de planta o toda una sección, que están elaboradas en vistas a conseguir un estado determinado de la ejecución de los trabajos, y pueden ser utilizados numerosas veces para la construcción de inmuebles del tipo considerado. Fijan la duración, el orden de sucesión y los procesos de ejecución de las distintas operaciones que incumben a cada puesto de trabajo. Una norma tecnológica-tipo está basada en los resultados de los cálculos técnicos y apropiados, efectuados de acuerdo con las disposiciones de las «Normas y evaluaciones unificadas».

La tipificación de la ejecución de los procesos tecnológicos según las técnicas modernas y con una mecanización integrada, desempeña un papel considerable en la normalización de la producción de la industria de la construcción. Para resolver este problema, se establecen fichas tecnológicastipo para todos los principales procesos previstos en las normas tecnológicas. Una ficha tecnológica lleva los siguientes puntos:

indicación de la estructura tecnológica del proceso y de las operaciones, así como del orden de su sucesión;

determinación de la técnica de ejecución del proceso: elección de los procesos de ejecución de los trabajos; determinación de los tipos y de las características de las máquinas, material, dispositivos e instalaciones que van a utilizarse;

definición de la organización del proceso en el tiempo y en el espacio, así como la organización del trabajo;

determinación de las cantidades indispensables de material, del equipo técnico y de la mano de obra;

cálculo de los índices de producción.

El método mencionado es, sin duda, la forma más eficaz de organización, ejecutada por empresas de construcción especializadas y equipadas con una maquinaria adecuada para la producción. La investigación ha mostrado que el sistema más racional de construcción de viviendas es el sistema continuo, complejo que engloba no solamente a la construcción de viviendas, sino también la preparación en obra, los trabajos de movimientos de tierra las vías de comunicación, los servicios, etc. La mecanización no solamente de las operaciones de producción, sino de la manutención y de las operaciones de transporte, desempeñan un papel esencial.

\section{La teoría básica de la construcción en cadena o el "flow-line method"}

El flow-line o producción en cadena es un sistema científico de construcción basado en premisas teóricas y es un medio, probado, de reducir el coste y la intensidad del trabajo de la edificación, incrementando la calidad.

La experiencia ha demostrado que en algunos países, por ejemplo, la Unión Soviética, el coste se reduce de un 6 a un 12 por 100 y la intensidad de trabajo de un 20 a un 30 por 100, mientras que la producción se incrementa de un 50 a un 100 por 100 . 
La producción en cadena exige:

- un abastecimiento ininterrumpido y continuo de materiales y componentes de edificios (estructuras, elementos y productos intermedios) por parte de las industrias de la construcción;

- un flujo ininterrumpido del proceso tecnológico en empresas de la industria de la construcción y las relacionadas con el transporte, maquinaria de construcción, utillaje y equipo;

— jornadas de cantidades uniformes de trabajo por unidad de tiempo;

- un rendimiento continuo e ininterrumpido de construcción (edificios, instalaciones y partes de los mismos).

La organización de una construcción en cadena basada en un rendimiento continuo tiene que tener en cuenta las siguientes medidas tecnológicas y de organización:

El proceso de producción debe ser separado en sus procesos componentes.

La mano de obra debe ser dividida entre las operaciones a realizar.

Debe ser creado un ritmo de producción.

El proceso tiene que coordinarse en el tiempo y en el espacio.

La producción continua en cadena es puesta en obra por organismos especializados de construcción de capacidad predeterminada, que disponen del material técnico apropiado, de su aprovisionamiento de materiales y otras necesidades. Dentro de los consorcios encargados de proceder a las construcciones de viviendas se han creado departamentos especializados en ciertas etapas de la producción (construcción de partes subterráneas de los edificios, levantamiento de la superestructura, trabajos de acabado, etc.). Por otra parte, hay otros organismos especializados en ciertos trabajos particulares, tales como "Santekhmontaje» (Instalaciones sanitarias y técnicas), "Derstroï» (vías de acceso), (Elektromontaje» (Instalaciones eléctricas), etc. En el seno de los organismos de construcción se prevé una intensa especialización muy intensa, que impulsa a la creación de equipos o de grupos especializados.

Un aprovisionamiento de materiales y de equipo técnico que sea a la vez completo y efectuado en tiempo útil representa una condición «sine qua non» para la producción en cadena. Cuando se construyen viviendas de un tipo determinado, inmuebles de grandes paneles, por ejemplo, la cantidad y la naturaleza de los lotes de materiales y de elementos de construcción tienen que ser invariables. En estas condiciones es indispensable crear empresas capaces de abastecer los artículos previstos por lotes completos. Estas empresas serán los complejos de construcción de inmuebles.

La producción continua estará organizada de forma que la capacidad de producción no oscile más que un mínimo durante los períodos en los que el flujo tecnológico se readapta en vistas de una nueva producción (un nuevo tipo de inmuebles) o durante los cuales se constituyen adelantos en los artículos semiacabados. La dispersión territorial de las obras no tiene más que ligeras repercusiones en los índices de producción de flujo continuo.

\section{Planificación de un flujo tecnológico continuo de larga duración}

La planificación de un flujo tecnológico de larga duración se funda en algunos principios importantes, que se citan a continuación:

la capacidad de producción debe quedar invariable, lo que hace que la intensidad de todos los flujos (elementales, especializados, de obra) debe ser constante;

se tiene que eliminar cualquier irregularidad o discontinuidad de la producción;

tođos los elementos del flujo tecnológico continuo deben estar equilibrađos por el establecimiento de un ritmo uniforme para la marcha de todos los flujos de larga duración.

Para elaborar un proyecto de organización de los trabajos según un flujo tecnológico continuo, es necesario disponer de un cierto número de datos iniciales, especialmente los que se refieren a las posibiliđađes de producción del organismo de construcción, a los programas de trabajo para el año y para más largo plazo (para los dos o tres próximos años), así como a las informaciones sobre las modalidades de los trabajos de construcción a efectuar. Los proyectos de organización de los trabajos según un flujo tecnológico continuo encierran el estudio de cinco grupos de cuestiones (ver el cuadro adjunto). 
CUESTIONES A CONSIDERAR EN UN PROYECTO DE ORGANIZACION DE LOS TRABAJOS SEGUN UN FLUJO TECNOLOGICO CONTINUO

Enumeración de las cuestiones

1. Volumen de los trabajos y naturaleza del programa de producción del organismo de construcción.

2. Estructura $e$ intensidad del flujo integrado, así como de los flujos de obra y de los especializados.

3. Duraciones y modalidades de puesta en obra del flujo integrado y de los flujos de obra y de los especializados sobre las obras previstas en el programa de producción.

4. Aprovisionamiento del proceso de los trabajos continuos en cadena en los medios de producción.

5. Estructura del organismo de construcción llamado a trabajar bajo el régimen de un flujo tecnológico continuo de larga duración.

Objetivos
Determinación del volumen de los
trabajos y de la naturaleza del pro-
grama de producción del organis-
mo de construcción. Elaboración
del programa de producción del
organismo de construcción y de
sus subdivisiones, teniendo en
cuenta las exigencias de un flujo
tecnológico continuo.

Determinación de la estructura y de la intensidad del flujo integrado, así como de los flujos de obra y de los especializados.

Determinación de las duraciones y modalidades de la puesta en obra de los diferentes flujos sobre las obras previstas en el programa de producción.

Determinación de las necesidades, en medios de producción, de los centros de aprovisionamiento y relativos a éstos, así como de las modalidades y de las épocas de sus entregas.

Determinación de la estructura del organismo de construcción llama. do a trabajar bajo el régimen de un flujo tecnológico continuo.

Soluciones
3
Estudio de los proyectos del arquit

Estudio de los proyectos del arquitecto y del constructor relativos a las obras previstas en el programa de producción y cálculo de los volúmenes, según los planos de ejecución.

Cálculo de la intensidad de los flu. jos.

Establecimiento de ciclogramas (gráficos de ejecución de las distintas obras y ciclogramas de recapitulación) relativos a los trabajos de construcción en cadena a efectuar en todas las obras previstas en el programa de producción del organismo de construcción.

Establecimiento de gráficos de aprovisionamiento en medios de producción, necesarios para ejecutar el programa de trabajo.

Determinación del número de subdivisiones del organismo de construcción y cálculo de su capacidad de producción, según la intensidad prevista para el flujo continuo integrado.

\section{La construcción continua en cadena para la construeción}

La experiencia ha demostrado que no siempre se han alcanzado los fines deseados usando construcciones en cadena a corto plazo para la construcción de grupos individuales de edificios. Estas construcciones en cadena a corto plazo no pueden sustancialmente influenciar la eficacia de trabajo en la organización de la construcción. La frecuente reorganización de la producción observada en estos casos tiende a declinar y es, por lo tanto, perjudicial. Desde luego, en Ucrania, con un año del empleo del método en cadena para la construcción de viviendas de cinco pisos, la utilización de la capacidad de producción en el método en cadena de una organización de construcción es de 82 por 100 para casas de grandes paneles, 76 por 100 para casas de grandes bloques y 60 por 100 para casas en ladrillo.

En los sectores de construcción a gran escala (viviendas, industrias, etc.) es preferible emplear el sistema continuo más eficiente de construcción en cadena extendiéndolo a cierto número de años. Las combinaciones de construcciones de viviendas están desempeñando en él un papel importante.

Las características especiales de la producción continua en cadena (larga duración, selección de los productos de acuerdo con una capacidad de producción predeterminada, producción en serie, especialización, estabilidad de los materiales y de los productos técnicos, nivel de standardización) determinan las perspectivas de uso y su futura extensión en la construcción. La teoría de la producción continua en cadena da solución a los siguientes problemas básicos:

- métodos de balance para calcular las proporciones de la construcción en cadena para proyectos primarios y especializados;

$\rightarrow$ medios para eliminar interrupciones en la producción;

- modos de reorganizar la estructura tecnológica de la construcción en cadena;

- investigación del volumen de producción tecnológica y establecimiento de los métodos para su cálculo. 


\section{La mecanización integrada en el cuadro de la construcción en cadena}

La principal característica de la construcción en gran escala de viviendas que se efectúa en Ucrania es la gran aceptación del sistema, que consiste en construir los edificios ensamblando elementos de grandes dimensiones prefabricados, utilizando métodos de trabajo en cadena con ayuda de una mecanización integrada. Esta representa la principal condición a cumplir para poner en práctica métodos de construcción industrializados.

Con una mecanización integrada, todas las operaciones del proceso de construcción, tanto las principales como las auxiliares, se efectúan con la ayuda de un conjunto de máquinas que son todas adaptadas unas a otras en sus parámetros o características y en su rendimiento. Los parámetros, el rendimiento y la disposición de las máquinas principales determinan la elección y la disposición de las máquinas complementarias.

Los principales índices de eficacia de una mecanización integrada son el consumo de mano de obra y el coste por unidad final de producción, así como el rendimiento (la producción) de cada puesto de trabajo en las máquinas.

Las principales condiciones que una mecanización integrada debe reunir para su máxima eficacia son las siguientes: hacer una elección racional de máquinas y lotes de máquinas y satisfacer todos los requisitos para asegurar una óptima utilización de los medios de mecanización.

La construcción en cadena, que está caracterizada por la regularidad y la continuidad de la producción, por un ritmo preciso y por un plan riguroso de los trabajos de ejecución, impone a las máquinas reunir un cierto número de condiciones particularmente en lo que concierne a su movilidad, su facilidad de transporte y el tiempo necesario para su puesta en marcha.

La construcción en cadena se presta mejor que cualquier otra a una explotación eficaz de las máquinas, y a un acrecentamiento máximo de su productividad. Primeramente, las máquinas y lotes de máquinas funcionan según un ritmo regular y bajo una carga uniforme; por otra parte, el montaje, el desmontaje y el desplazamiento de las máquinas de una obra a otra, las medidas profilácticas, el entretenimiento y las reparaciones corrientes se realizan según un horario estrictamente establecido.

Cuando se construye un barrio residencial o un grupo de viviendas por el método del trabajo en cadena, es necesario organizar un flujo tecnológico integrado compuesto de varios flujos especializados o de obra. Estos flujos engloban la integridad de los trabajos de construcción y de montaje, preparación técnica del terreno; eđificación de viviendas, de edificios públicos, comunitarios, culturales y de diversión; y dotación de los elementos de confort.

La elección y el cálculo de los medios mecánicos necesarios se hacen desđe el principio al establecer la planificación del trabajo. La unidad de cálculo es el flujo elemental, con el lote de máquinas tecnológicamente necesarias para ponerlo en obra. Este lote elemental de máquinas tiene una capacidad de rendimiento determinada. Para poner en obra los flujos especializados y de obra, se toma un cierto número de lotes elementales. De esta manera es superfluo calcular en cada caso particular las necesidades en máquinas de tal o cual categoría, en función de la intensidad de flujos. En lugar de proceder a estos cálculos, se determina la cantidad necesaria de lotes completos. La determinación de los lotes óptimos de máquinas de construcción de carreteras se efectúa por los métodos usuales, fundados en la comparación de los índices técnicos y económicos.

Si la cantidad necesaria de máquinas de construcción se calcula en función del volumen anual de los trabajos de construcción y de montaje, de las normas de rendimiento material en volumen de máquinas, y de varios factores distintos, este método no conviene para las máquinas de acabado y los útiles mecánicos. Sólo las máquinas de grande y mediana potencia pueden ser determinadas según su rendimiento. En lo que se refiere a los cálculos que afectan a las máquinas pequeñas de acabado, es recomendable proceder considerando los lotes tecnológicamente indispensables para poner en obra un flujo elemental o para mecanizar un equipo especializado de obreros propuestos para los trabajos de acabado (pintores, ebanistas, etc.).

Una vez finalizadas las actuaciones de los ponentes, se inició una animada discusión en la cual diferentes oradores destacaron la interdependencia entre la continuidad de la demanda y la continuidad técnica, puestas en relieve anteriormente, y la necesidad de desarrollar métodos adecuados para prever la demanda y evaluar la máxima capacidad productiva de la industria de cualquier país. Todos ellos estuvieron de acuerdo en que una de las características principales de la industria de la construcción es que su capacidad de producción no puede ser almacenada: el problema consiste, pues, en encontrar los medios adecuados para mantener el flujo tecnológico requerido en una producción continua, a pesar de la discontinuidad de la demanda en el tiempo.

Este problema, en las economías de mercado libre consiste a menudo en encontrar una fórmula adecuada para la acción del gobierno, de forma que éste pueda restringir el exceso de la demanđa para las nuevas construcciones cuando la capacidad del sector de construcción sea insuficiente para satisfacer la demanda al nivel existente entre los precios y las viviendas. El problema se encuentra complicado por cuestiones de equilibrio entre las inversiones y los ahorros a fin de evitar la inflación, y por cuestiones sociales dírigidas por la política del gobierno en relación con el control de viviendas. 


\section{Le Gomgrabs die Proguge}

Fernando Aguirre de Yraola, Dr. Architecte.

Dans cet article l'auteur commente deux des thèmes traités au cours du Symposium tenu à Prague, au sujet de l'évolution de la structure de l'industrie du bâtiment, dont les titres sont: «L'évolution des rapports entre les diverses personnes intervenant dans le bâtiment», et "La continuité de la demande et la continuité technique de la production». Les rapports commentés furent développés par les délégués du Danemark, de Pologne, des Pays-Bas et d’Ukraine. Quelques documents auxiliaires, rédigés et commentés dans les réunions par les délégués d'autres pays, ont été également très utiles pour l'étude et la discussion des points traités de grand intérêt. L'analyse de la méthode de production à la chaine, ou «Flow-line method», présente un intérêt tout spécial.

\section{The Proguge Eomgmess}

Fernando Aguirre de Yraola, Dr. architect.

This article is a commentary of the matters discussed at the Prague Seminar on the evolution of the construction industry, of which the detailed headings are «Evolution of the relationship of the various people who take part in the construction industry" and «The continuity of demand and the technical continuity of production». The papers discussed were submitted by the delegates of Denmark, Poland, Holland and Ucrania. The study and ellaboration of the main themes was greatly aided by a number of auxiliary documents, submitted and discussed by the delegates of other countries. The analysis of production line and flow line methods was of outstanding interest.

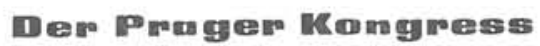

Fernando Aguirre de Yraola, Dr. Architekt.

In diesem Artikel werden zwei Themen betrachtet, die auf dem Prager Kongress über die Entwicklung der Struktur in der Bauindustrie behandelt wurden, und zwar: «Die Entwicklung der Beziehungen zwischen den einzelnen an einem Bau beteiligten Personen» und «die Kontinuierlichkeit in der Nachfrage und in der Produktion». Es sprachen die Vertreter Dänemarks, Polens, der Niederlande un der Ukraine. Von gleichem interesse für das Studium und die nachfolgende Diskussion der interessanten Themen waren einige Unterlagen, die von Vertretern anderer Länder abgefasst und kommentiert wurden. Die Analyse der Methode der Kettenproduktion, die «Flow-line-method», erweckte ebenso grosses Interesse. 